\title{
Analysis of Linux Scheduling with VAMPIR
}

\author{
Michael Kluge and Wolfgang E. Nagel \\ Technische Universität Dresden, Dresden, Germany \\ \{Michael.Kluge, Wolfgang. Nagel\}@tu-dresden.de
}

\begin{abstract}
Analyzing the scheduling behavior of an operating system becomes more and more interesting because multichip mainboards and Multi-Core CPUs are available for a wide variety of computer systems. Those system can range from a few CPU cores to thousands of cores. Up to now there is no tool available to visualize the scheduling behavior of a system running Linux. The Linux Kernel has an unique implementation of threads, each thread is treated as a process. In order to be able to analyze scheduling events within the kernel we have developed a method to dump all information needed to analyze process switches between CPUs into files. These data will then be analyzed using the VAMPIR tool. Traditional VAMPIR displays will be reused to visualize scheduling events. This approach allows to follow processes as they switch between CPUs as well as gathering statistical data, for example the the number of process switches.
\end{abstract}

\section{Introduction}

The VAMPIR [7] tool is widely used to analyze the behavior of parallel (MPI, OpenMP and pthreads) as well as sequential programs. This paper will demonstrate how the capabilities of VAMPIR can be used to analyze scheduling events within the Linux kernel. These events are gathered by a Linux kernel module that has been developed by the authors. This development has been motivated by an scheduling problem of an OpenMP program that will be used within this paper to demonstrate the application of the software.

Linux itself is an operating system with growing market share in the HPC environment. Linux has its own way of implementing threads. A thread is not more than a process that shares some data with other processes. Within the Linux kernel there is no distinction between a thread and a process. Each thread also has its own process descriptor. So within this paper the terms 'thread' and 'process' do not differ much. Although we will talk about OpenMP threads, those threads are also handled by the Linux Kernel as normal processes when we are talking about scheduling.

The first section gives an short overview about the state of the art in monitoring the Linux kernel. The next section is dedicated to our Linux kernel module and the output to OTF [1]. Within the third section will show how various VAMPIR displays that have been designed to analyze time lines of parallel programs or messages in MPI programs can be reused for an visual analysis of Linux scheduling events. This paper is closed by a short summary and an outlook. 


\section{Analyzing Scheduling Events in the Linux Kernel}

Analyzing scheduling events is an interesting piece within the whole field of performance analysis due to effects that can be traced back to a specific process placement or cache thrashing. Within this paper we are referring to a multiprogramming environment. This means that multiple programs do run in parallel on a given set of CPUs. The processes associated to these programs are not pinned to a specific CPU. Therefore the scheduler is free to place the processes as needed onto available CPUs.

We have identified two main approaches to analyze the scheduling behavior of an specific system. The first idea is to instrument the kernel scheduler itself to monitor its actions. This would have the advantage of having insight into scheduler decisions. An other idea is an indirect approach. If the CPU number that process is running on over time is traced as well as information about the process state (running or suspended), the priority, the nice value, interactivity etc. one can show strength and weaknesses within the scheduler also. All the information needed for the second approach are available within the process descriptor in the Linux kernel. The information needed for the first approach is only locally available only within the scheduler implementation and not globally in the kernel. Opposite to that, the list of current tasks and their properties are available everywhere within the kernel.

There is no exiting tool we have found that is able to gather those information described above. The Linux Trace Toolkit [5] collects information about processes but does not have any information about the CPU number a process is running on. There are tools that are able to instrument a kernel (like KernInst [11] or KTau [8]) that require a unique program to be written and put into the kernel. Monitoring the /proc file system [9] would be an solution but cannot provide the fine granularity needed. For AIX the AIX kernel trace facilities can be used to gather data about various events in the kernel [2].

For really fine grained monitoring of the process-to-CPU mapping and the process state we decided to try a different way.

\section{Tracing Scheduling Events}

Our approach utilizes the second idea from the section above. Because the information about all tasks on the system are available at each point in the Linux kernel, the main idea is to write a kernel module that dumps the information needed at specifiable time intervals. Some kind of GUI or automatic analysis tool could later be used to analyze this data. The advantage of a kernel module is the ability to load and unload the module as needed as well as the short time for recompilation after a change in the source code because the kernel itself is not being touched [4].

The design of the kernel module is as follows. A kernel thread is created and inspects all given threads at an adjustable time interval. The minimum time between two inspections is the so called 'kernel frequency' which can be chosen 
at the kernel setup with 100, 250 or 1000 ticks per second. The kernel module is given a particular process id (PID) to watch. It will inspect this PID and all its children and will dump all needed information (actually CPU and process state) to the relayfs [12] interface. This way the user is able to select all processes or any part of the current process tree. On problem here are processes that get reparented. If a process finishes that still has child processes, those child processes will get the init process (1) as parent. If not all processes but a specific subset is traced, this child processes will vanish from the trace at this point in time.

The kernel module itself can be started, configured and stopped via an interface that has been made available through the sysfs file system. So the kernel module can stay within the kernel without generating overhead when nothing needs to be measured.

To dump the data gathered to the disk, a relatively new part of the Linux kernel, relayfs, is used. relayfs is an virtual file systems that has been designed for an efficient transfer of large amounts of data from the kernel to user space. It uses one thread per CPU to collect data through a kernel wide interface and to transfer the data. The data is collected inside relayfs within sub buffers. Only full sub buffers are transfered to the user space. On the user side, one thread per CPU is running to collect the full sub buffers and to write the data to a file (one file per CPU). This thread is sleeping until it gets a signal from the kernel that a full sub buffers is available. This approach is scalable and disturbs the measured tasks as less as possible.

In summary the kernel module currently supports the following features:

- enable/disable tracing from the user space on demand

- tracing of user selectable processes or tracing the whole system

- changing parameter settings from the user space (via sysfs)

\section{Using VAMPIR to Analyze Scheduling Events}

Now we have a collection of events that describes which process has been on which CPU in which state in the system at different timestamps. The amount of data can become very large and needs a tool to be analyzed. Some kind of visual and/or automatic analysis is needed here. There are basically two different things that we want to analyze from those trace files:

1. number of active processes on each CPU

2. following the different processes (and their current states) on the CPUs over the time

As threads and processes are basically treated the same way by the Linux kernel, the hierarchical structure between all processes/threads is also known at this point. For the first application it is possible to count the tasks in the state 'runnable' on each CPU. To actually be able to view this data the following approach have been identified: 
- each CPU is mapped to what VAMPIR recognizes as a process

- task switches can be show as an one byte message between the associated CPUs (processes)

- forks and joins can also be shown as messages

- the number of forks, joins and task switches per kernel tick are put into counters

By using different message tags for the forks, joins and task switch different colors can be used within VAMPIR to make the display even more clear. The filter facilities of VAMPIR can be used to analyze CPU switches, forks or joins independently. Due to the zooming feature of VAMPIR (which updates each open display to the actual portion of the time line that is selected) it is possible to analyze the scheduling behavior over time.

On the beginning all CPUs (processes for VAMPIR) do enter a function called '0'. When the first process is scheduled onto a CPU is will leave this function and enter a function called '1'. By following this idea we can have a very informative display about the number of runnable processes on the different CPUs. By looking at VAMPIR's time line and the counter time line in parallel we already get a good feeling on what was happening on the system.

For following the processes over different CPUs this scheme needs to be extended not only to have one VAMPIR process line for a CPU but to have multiple process lines per CPU where the real processes will be placed on. Those lines will be called a stream on that CPU from now. In this scenario, processes that enter a specific CPU will be placed on a free stream. So for each process one or two virtual function were defined for VAMPIR. One is always needed and denotes that on one stream a specific process ID is present. This can further be extended to have distinct virtual VAMPIR functions for the two states of a process (running/not running). In the second case we can generate a leave event for one virtual function and an enter event to the other virtual function on the same stream when a process switches its state.

The idea of modeling task switches as messages allows to use VAMPIR's Message Statistics window to analyze how many processes switched from one $\mathrm{CPU}$ to another and how often this took place for each CPU (from-to) pair.

\section{OTF Converter}

To be able to analyze the collected data with VAMPIR a tool is needed to convert the data dumped by the kernel module to a trace file. We have chosen to utilize the OTF library due to its easy handling. Within relayfs the data obtained by the kernel thread are dumped to the file that is associated with the CPU where the kernel thread is running on at this point in time. The converter has been written to serialize all events within these files after the program run and to follow the tasks when they jump between the CPUs. It generates OTF output with all necessary information like process names and state, CPU utilization together with various counters. 
The example we will look at within the next section creates about $1 \mathrm{~GB}$ of trace data together from all CPUs. This example runs for about 6 minutes on 8 CPUs. The conversation to the OTF file format takes about one minute and results in OTF files between 100 and $120 \mathrm{MB}$.

\section{Example}

Our example is derived from a problem observed on our Intel Montecito test system. It has 4 Dual Core Itanium 2 CPUs running at $1.5 \mathrm{GHz}$ (MT disabled). The multiprogramming capabilities of a similar system (SGI Altix 3700) have been investigated with the PARbench Tool [6], [3], [10]. One result here has been that an OpenMP parallelized program that is doing independent computation in all threads all the time (without accessing the memory) is behaving unexpected hin an overload situation. We put eight sequential tasks and eight parallel tasks (which open eight OpenMP threads each) on eight CPUs. So we have 72 active threads that all need CPU time and do hardly any memory access. The algorithm used on each tasks is the repeated (100000 times) calculation of 10000 Fibonacci numbers. The sequential version takes about 2 seconds to run. The OpenMP parallel program exists in two flavors. The first flavor has one big parallel section, $100000 * 10000$ numbers are calculated in one block. The second implementation opens and closes the OpenMP parallel section 100000 times to calculate 10000 Fibonacci numbers. One parallel task with 8 parallel threads also needs 2 seconds for both flavors. In the overload situation, all 72 threads did run concurrently on the system. If we used the first OpenMP implementation all 72 tasks/threads

Table 1. Wall time in seconds of the sequential and parallel program version in different execution environments

\begin{tabular}{|l|r|r|r|}
\hline program & big OpenMP block & \multicolumn{2}{|c|}{$\begin{array}{c}\text { small OpenMP block } \\
\text { busy waiting }\end{array}$} \\
& & yield CPU \\
\hline \hline sequential & $19-21$ & $2-3$ & $8-16$ \\
\hline parallel & $19-21$ & $45-50$ & $21-23$ \\
\hline
\end{tabular}

exited after about 20 seconds (+/- 1 second). If we use the second flavor, the eight sequential tasks exit after 2 to 3 seconds and the parallel tasks exit after 45 to 50 seconds.

The explanation for the different behavior we found after the investigation with our tool. It is the fact, that for the first flavor the tasks do not synchronize. On an overloaded system the tasks get out of sync easily. The default behavior of the OpenMP implementation for a synchronization point is a busy wait for $200 \mathrm{~ms}$ and a call to sleep () afterwards. That way the OpenMP threads for the first flavor do synchronize just once and they use their full timeslice to do calculation. In the second flavor the parallel task spend part of their time slice with busy waiting. By putting the busy waiting time to 0 by using export KMP_BLOCKTIME=0 


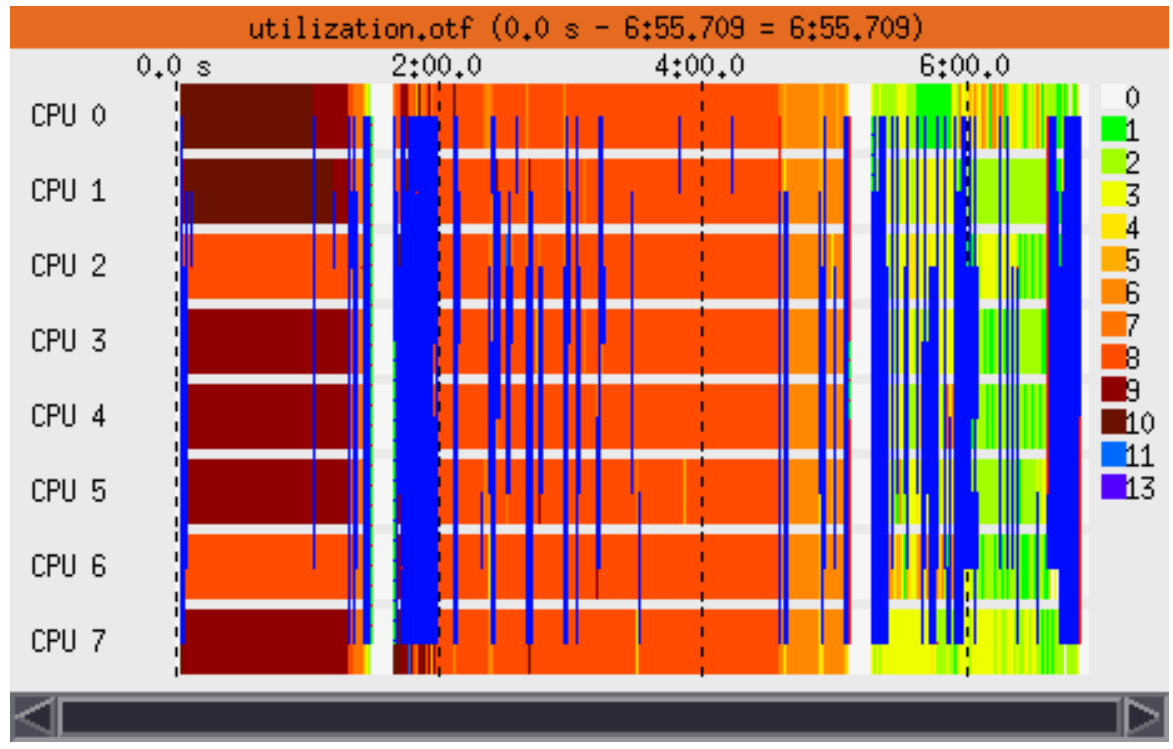

Fig. 1. Screenshot of the full time line, note the three different phases

this can be improved. The sequential tasks exit after 8 to 16 seconds and the parallel tasks need between 21 and 23 seconds. The numbers are compiled in table 1.

The VAMPIR screenshot for the scheduling time line for all three runs is given in Figure 1. All switches for a task from one CPU to another is marked by a (blue) line. From the beginning of the time line to about 1:50 min the run for the one big OpenMP block has taken place. Afterwards the OpenMP busy waiting example is executed. As the last example from about 5:30 minutes to the end of the time line the run with disabled busy waiting is shown. Figure 2 shows all switches from/to all CPUs. By zooming in and looking into the different parts of the time line, the following facts could be collected for the three different runs:

1. After spawning all the processes the system is balanced after a relatively short period of time. The load on the individual CPUs is well balanced. Almost no rescheduling occurs during this period of time.

2. For the second run the balancing of the system takes much longer. During the whole second run every few seconds there are some scheduling events where tasks switch between CPUs. The reason for this is that some tasks get suspended (after the busy wait time has elapsed) and the system needs to be re-balanced afterwards.

3. The third case again is very different. Tasks get suspended very often and awakened thus the CPU utilization jitters a lot (due to the short OpenMP regions and no busy waiting). For that reason the system never gets well balanced but due to the fact that there are no CPU cycles spent busy waiting this scenario has a shorter wall time than the second one. 


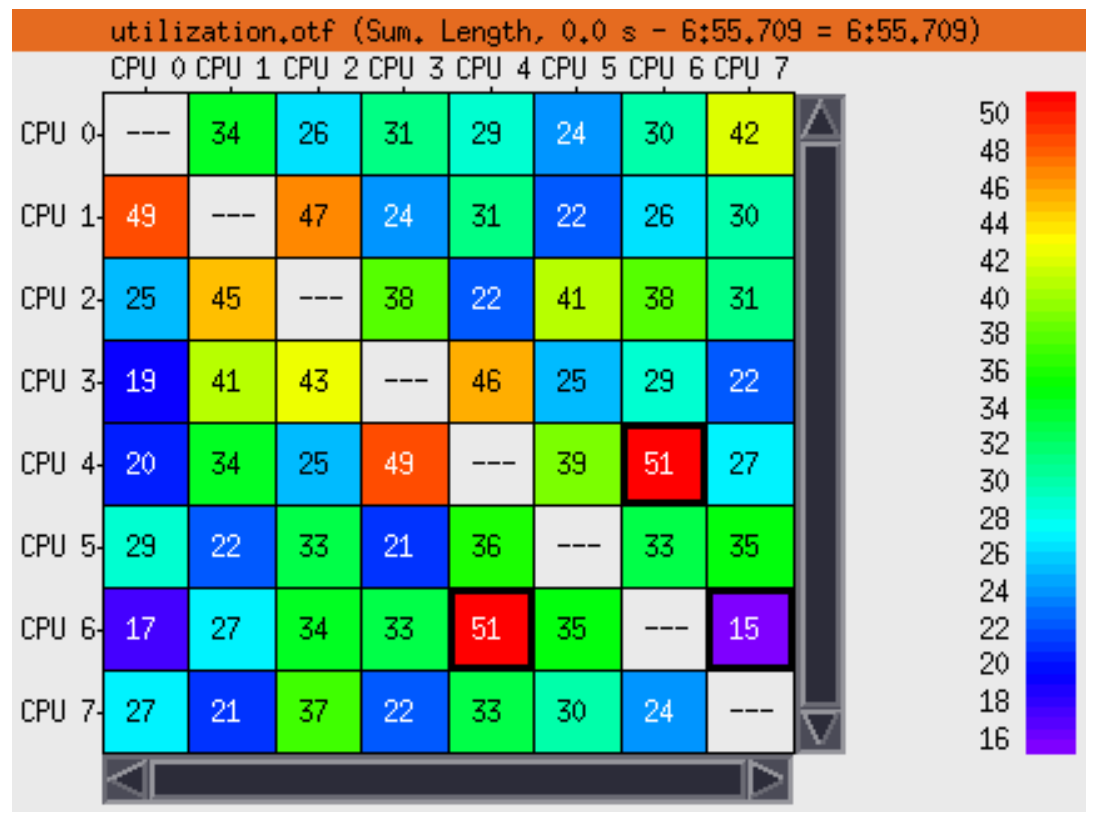

Fig. 2. Screenshot of all process switches

\section{Conclusion}

The work presented has two main results. First of all we designed a convenient measurement environment to collect scheduling events from the Linux kernel (a kernel module + relayfs). And we reused VAMPIR's capabilities for a different purpose. Traditional displays from VAMPIR have been reinterpreted for our purposes and do provide very useful information to analyze the scheduling behavior of a Linux system. A test case has been investigated and the underlying problem has been identified.

For the future there are various opportunities to follow. One very interesting idea is to correlate this information with a traditional program trace to be able to follow effect like cache thrashing or other things that are only analyzable by looking at the whole system and not only looking onto a single program trace obtained in user space.

This work has also shown that short OpenMP sections in an overload situation on Linux is counterproductive. With busy waiting disabled this can be improved. This way the OpenMP threads do sleep while waiting on a barrier. For this there is a possibility that the Linux kernel classifies this threads as 'interactive' and starts to shorten their timeslice.

The authors wants to thank her colleges Andreas Knüpfer, Holger Brunst, Guido Juckeland and Matthias Jurenz for useful discussions and a lot of ideas. 


\section{References}

1. Andreas Knüpfer, Ronny Brendel, Holger Brunst, Hartmut Mix, and Wolfgang E. Nagel. Introducing the Open Trace Format (OTF). In Vassil N. Alexandrov, Geert Dick van Albada, Peter M.A. Sloot, Jack Dongarra, Eds., Computational Science - ICCS 2006: 6th International Conference, Reading, UK, May 28-31, 2006. Proceedings, volume II of Lecture Notes in Computer Science. Springer Berlin / Heidelberg.

2. IBM. http://publib16.boulder.ibm.com/doc_link/en_US/a_doc_lib/aixprggd /genprogc/trace_facility.htm.

3. M.A. Linn. Eine Programmierumgebung zur Messung der wechselseitigen Einflusse von Hintergrundlast und parallelem Programm. Technical Report Jül-2416, Forschungszentrum Jülich, 1990.

4. Robert Love. Linux Kernel Development (german translation). Number ISBN 3-8273-2247-2. ADDISON-WESLEY, 1 edition, 2005.

5. Mathieu Desnoyers and Michel R. Dagenais. Low Disturbancea Embedded System Tracing with Linux Trace Toolkit Next Generation. http://ltt.polymtl.ca, November 2006.

6. W.E. Nagel. Performance evaluation of multitasking in a multiprogramming environment. Technical Report KF-ZAM-IB-9004, Forschungszentrum Jülich, 1990.

7. Wolfgang E. Nagel, Alfred Arnold, Michael Weber, Hans-Christian Hoppe, and Karl Solchenbach. VAMPIR: Visualization and Analysis of MPI Resources. In Supercomputer 63, Volume XII, Number 1, pages 69-80, 1996.

8. A. Nataraj, A. Malony, A. Morris, and S. Shende. Early Experiences with KTAU on the IBM BG/L. In Proceedings og EUROPAR 2006 Conference, LNCS 4128, pages 99-110. Springer, 2006.

9. redhat Documentation. http://www.redhat.com/docs/manuals/linux/RHL-7.3Manual/ref-guide/ch-proc.html, November 2006.

10. Rick Janda. SGI Altix: Auswertung des Laufzeitverhaltens mit neuen PARBenchKomponenten. Diplomarbeit, Technische Universität Dresden, June 2006.

11. Ariel Tamches and Barton P. Miller. Using dynamic kernel instrumentation for kernel and application tuning. In International Journal of High-Performance and Applications 13, 3, 1999.

12. Tom Zanussi et.al. relayfs home page. http://relayfs.sourceforge.net, November 2006. 